\title{
ASSOCIATION BETWEEN THE RISK FACTOR FOR ANTERIOR GLENOHUMERAL INSTABILITY AND BONE LOSS IN ACTIVE SPORT PLAYERS
}

\author{
Svetoslav Dobrilov, Maxim Zagorov, Kalin Mihov \\ Department of Orthopedics and Traumatology, University Hospital „St. Marina“ Varna
}

\begin{abstract}
INTRODUCTION: Practicing mass and professional sport is with growing popularity among young people. Sports like football, basketball, volleyball, and contact disciplines- boxing, martial arts , etc. are highly connected to trauma. Shoulder dislocation is very frequent, which in most of the cases turns into instability. The main cause for it is glenoid and/or humeral bone loss.

MATERIAL AND METHODS: For the period 2010- 2015 in the University Hospital "St.Marina" there were operated 22 patients with high physical activity- 5 of them were professional athletes. The volume of bone loss is measured with CT and with method proposed by Sugaya. The indications for operative treatment are based on history, clinical exam, imaging and ISIS score. The operative treatment performed is coracoid transfer over glenoid rim.

RESULTS: All patients had significant, for instability, bone loss. The postoperative period was smooth and there were no instability recurrences detected for the follow-up period.

CONCLUSIONS: Glenohumeral instability in athletes is a result of recurrent shoulder dislocations, the main cause for which is glenoid and/or humeral bone loss. Detecting and measuring the level of this bone loss is of great importance to the correct treatment algorithm, leading to joint stability and return to active and competitive sports.
\end{abstract}

Keywords: Glenohumeral instability, glenoid, bone loss, ISIS score

\section{INTRODUCTION}

Contact and overhead sports (basketball, volleyball, baseball (USA), fitness) are characterized with prolonged overuse of the upper extremities in end ranges of motion. This causes great stress to the joint and its stabilizing structures.

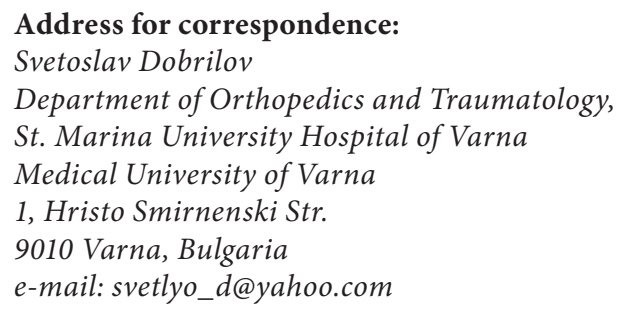

Received: July 24, 2015

Accepted: August 21, 2015
Glenohumeral instability is the inability of the humeral head to stay centered into the glenoid fossa and clinically it is manifested with excessive translation of the humeral head on the glenoid in rotation. This abnormal joint movement is presented with pain, dislocation or subluxation. The most important causes for this are traumatic dislocation, pathological changes (presence /absence of bone defects) and patient's age during first dislocation.

The diagnostics and treatment algorithm in patients practicing risk disciplines requires detection and evaluation of bone loss and its compensation.

\section{MATERIAL AND METHODS}

For the period 2010-2015 in Department of Orthopedics and Traumatology in the University Hospital "St. Marina" there were operated 22 male pa- 
tients. The mean age when the treatment begun was 25.4 yrs. The youngest one was 18 years old and oldest was 35 years, and the lateralization left: right was 9:13. All patients were with high physical activity and practicing risk disciplines, 5 of them were professional players. Of great importance is the history of the trauma/dislocation itself, the date of the first/last dislocation, the total count in the last 6 months, lateralization, etc.

All patients had a direct mechanism of dislocation. During the time of the first episode the patients under 20 years are $1(4,55 \%)$; 20 -30 yrs. - 20 (90,9\%) and above 35 yrs. - 1 (4,55\%) patient. The total number of dislocations/recurrences in one year before exam: under 4 - four patients $(18,2 \%), 4-6$ dislocations - 12 (54,5\%) and above 6 - six patients (27,3\%).

The clinical exam includes palpation, range of motion (ROM), strength of the upper extremity, sensory deficit (incl. proprioreception), reflex evaluation and special test for glenohumeral instability. It is recommended to examine the neighboring joints as well in order to exclude coexisting pathology. Because of that fact, instability is defined as a condition in which the humeral head is unable to stay centered in the glenoid fossa, it is important for the patient ing to dislocate". This is a leading symptom for possible pathology.

Stress-tests are used to evaluate shoulder ability to resist forces that influence stability in positions in which tense ligaments physiologically. This happens when the examiner puts the patient's glenohumeral joint in a position that causes subluxation /dislocation, pain or a feeling of "apprehension" or falling, so that he loses control over his shoulder. These tests are anterior apprehension test (11); anterior /posterior drawer test (8), hiperabduction test.(7)

After the exam, we continue with the imaging diagnostics. This includes:

X-ray ( AP, lateral, Bernageau view (2), axillary view)

CT with digital subtraction of the humeral head

\section{Contrast MRI}

When we have the clinical and X- ray data, if there are suspicions of bone loss we perform a primary CT protocol. If the symptoms are leading to a softtissue trauma we prefer arthro-MRI.

The most frequent sign, that we have seen on the images was the glenoid type "inverted pear" (fig. $1)$.
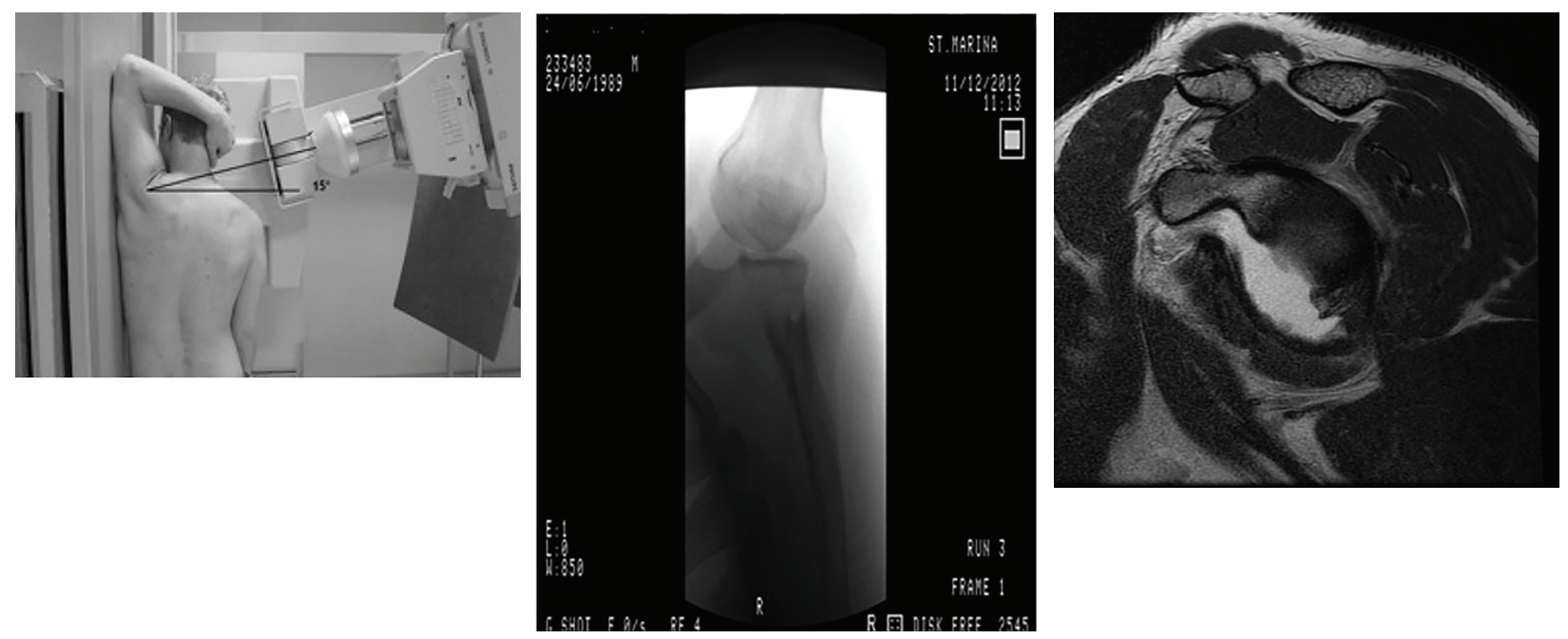

Fig. 1. Bernageau view - patient position (left 1-1) and

X-ray image (middle 1-2) and glenoid type "inverted pear" (right 1-3)

to demonstrate (if able) his functional problem. We asked the patient to put the arm in a position which is causing him pain and/or instability. In these cases the patients report a feeling of "my shoulder is go-
Our diagnostic algorithm includes the socalled ISIS (Instability Severity Index Score). When we have a score of under 6 pt., the patient is indicated 
for arthroscopic stabilization, but if score is equal or over 6 pt. -the solution is bony procedure.

\section{ISIS-Instability Severity Index Score}

Age under 20 years (2pt.)

Hill-Sachs, X-ray ER (2pt.)

Contact /overhead sports (1pt.)

Professional sport (2pt.)

Loss of anterior-inferior border of the glenoid (2pt.)

Hyperlaxity (1pt.)

If ISIS $\geq 6$ - there is $70 \%$ of a recurrence risk if a soft-tissue procedure was performed, when ISIS $<3$ - there is $10 \% .(4)$

A presence of bone loss (glenoid and/or humeral head), which is contributing to ISIS with 4 points, is detected and measured with CT/arthro MRI. We use a method proposed by Sugaya, which we consider to be most useful and reliable (fig. 2).

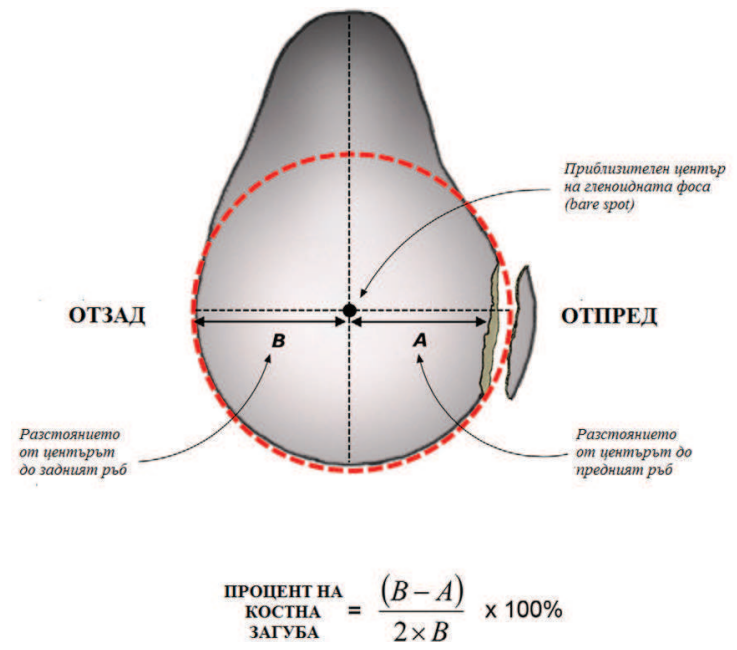

The average measured bone loss of the glenoid rim was $24.7 \%$ (19\% - 30\%). In 16 patients there was coexisting bone loss of the humeral head (HillSachs), which was greater than the glenoid surface (the so-called off-track defect). These aggregate defects are with a higher risk for instability and are indicated for bony substitution. Intraoperative findings confirm that, and the correlation between the size of the defects and the number of dislocations. History data, imaging, bone loss calculation and intraoperative pathological findings are confirming the relation between the time of the first dislocation, the frequency of recurrence and the level of bone loss. The younger the patient is, during their first dislocation, the greater the bone loss is $(6,13)$.

The ISIS score is an important component in our treatment algorithm. All patients in our group had ISIS $\geq 6$ points, which is an indication for a bony procedure, for substituting a bony defect and stabi-

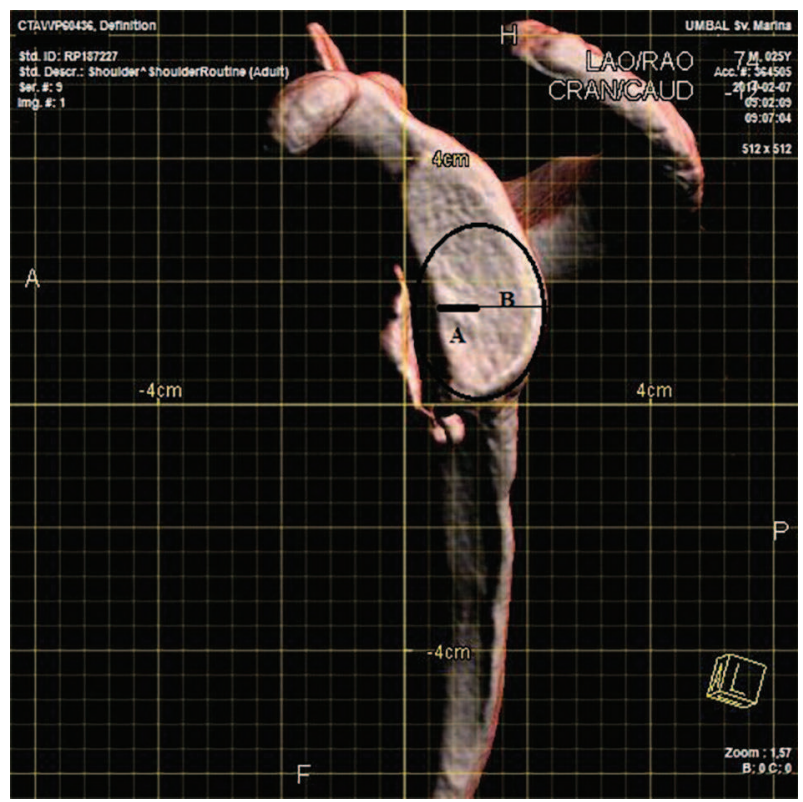

Fig. 2. Bone loss measurement as a percentage from the distances from the center of the glenoid to both ends. On the CT/ MRI image we define the crossing point of the transverse and longitudinal axis of the glenoid, which is its actual center. Distances from the center to the anterior $(\boldsymbol{A})$ and to the posterior $(\boldsymbol{B})$ are measured and the level of bone loss is calculated

There are a few more ways to calculate bone loss: the method of Husmans; the Pico-method; the overlapping method of Chang, etc. All methods give close findings but with more a complicated methodology. lizing the shoulder joint. We prefer a mini-open coracoids transfer (Latarjet operation). This is a resection of the coracoid process of the scapula with muscles attached to it and transferring it to the glenoid rim. This fills the defect and stabilizes the glenohu- 
meral joint. Correct graft orientation is essential for achieving a congruent and stable joint, especially in abduction and external rotation.

In operated patients we had Rowe score -excellent $70 \%$ (fig. 3), very good $16 \%$ with average limitation of external rotation of $7^{\circ}$. Three of the professional players returned to full sport activity by the 18-th week and there was no recurrence of instability. We had only one superficial infection, which was treated with a short antibiotic course.

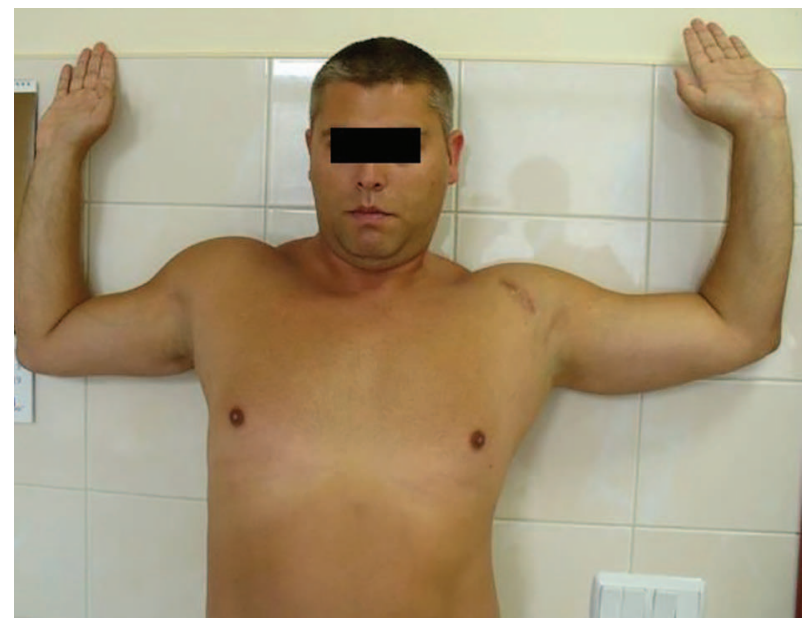

Fig. 3. ???

\section{DISCUSSION}

Pathological findings associated with glenohumeral instability could be capsule-labral (Bankar-lesion; SLAP-lesion, IGHL) and bony (Bony Bankart; Hill-Sachs). Frequency of the bipolar bone defect after a traumatic dislocation is from $8-95 \%$ for Bony Bankart and 25-100\% for Hill-Sachs lesion (15). Patients under 20 years of age have an $80 \%$ risk of bone damage and instability, for patients from 20-40 years of age the risk decreases to $60 \%$ and drops to $15 \%$ after 40 years of age (14).

Sugaya et al. define during arthroscopy and CT only $10 \%$ of the normal/ intact joints, $40 \%$ with erosive-compression defects of the glenoid and 50\% with Bony Bankart (16). Itoi et al. find that, lesion extending to $21 \%$ from glenoid length decreases with $50 \%$ stability of the joint, which corresponds to $18 \%$ of the glenoid deficiency of X-ray findings (West Point view) and $50 \%$ loss of glenoid debt on CT $(18,9)$. This percentage presents the so-called "critical point", over which, the glenohumeral joint is highly unstable in abduction and external rotation. Bony defects over $26 \%$ of glenoid width and $20 \%$ of length are decreasing stability substantially and are a cause for compromised soft-tissue stabilization $(16,10,18)$. Itoi et al. introduce the "glenoid track concept" (17). With 3-D
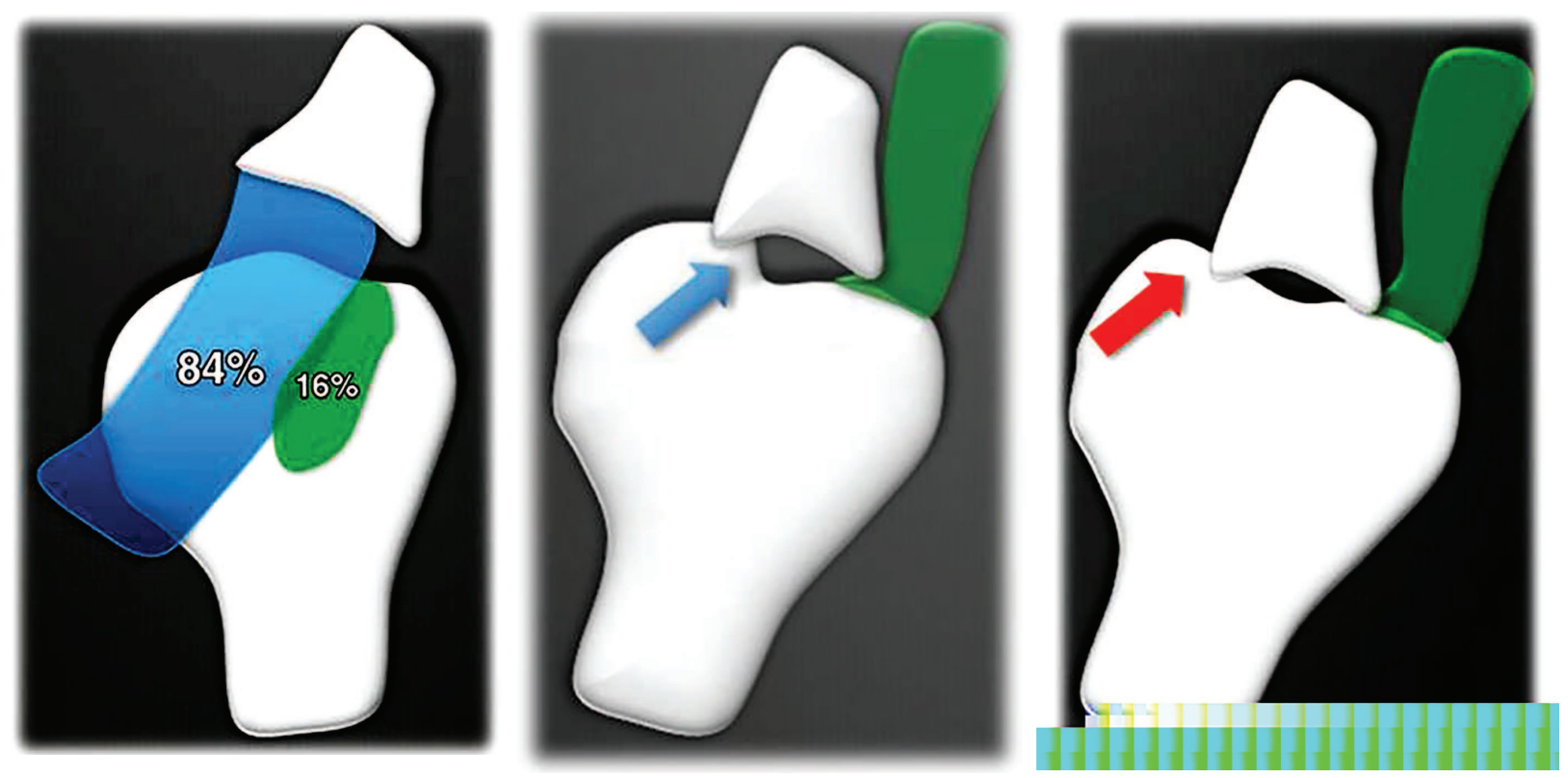

Fig. 4. "Glenoid track concept": left (4-1) size and localization of the glenoid track; a middle (4-2) Hill-Sachs defect is located into the "glenoid track". If there is a glenoid defect (GD) the Hill-Sachs lesion overlaps the glenoid and becomes offtrack (right 4-3) 
CT are identified bipolar bony defects and their relation to the different positions of the upper extremity. In abduction, the glenoid contact zone is moving from inferior-medial to superior-lateral, forming the so-called "glenoid track". Intact bony surface guarantees stability. Distance from the medial glenoid rim of the contact zone to the medial edge of the rotator cuff (glenoid track) is around $18 \mathrm{~mm}$, or $84 \%$ of the glenoid width in $60^{\circ}$ of abduction (17). If the Hill-Sachs defect is localized into the "glenoid track", there is no possibility for "entrapment" to the glenoid rim (non-engaging / off-track Hill-Sachs and there is no risk for instability. When the defect is located more medially (away from "glenoid track), there is a risk of entrapment to the glenoid rim- respectively instability. Such defect is defined as engaging/ offtrack Hill-Sachs (10) (fig. 4). Defining one defect as on/off-track happens with a CT scan (fig. 4)

\section{CONCLUSIONS}

Collision and overhead sports, especially those with a professional engagement present a risk for shoulder joint trauma. The frequency of shoulder dislocations is directly related to the volume of bone loss and it is highly dependable on the patient's age and the level of sports activity. Defining the risk factors for instability in active athletes is extremely important for the accurate diagnosis and treatment. This is a prerequisite for full and fast recovery and returning to active sport with a stable glenohumeral joint.

\section{REFERENCES}

1. Allain et. al. Long term results of the Latarjet procedure for the treatment of anterior instability of the shoulder. Am J Sports Med 1998

2. Bernageau J, Patte D, Debeyre J, Ferrane J. Value of the glenoid profil in recurrent luxations of the shoulder [in French]. Rev Chir Orthop Reparatrice Appar Mot 1976;62:142-7.

3. Bigliani LU, Newton PM, Steinmann SP et al (1998) Glenoid rim lesions associated with recurrent anterior dislocation of the shoulder. Am J Sports Med 26:41-455

4. Boileau P, Balg F. Risk factors for recurrence of shoulder instability after arthroscopic Bankart repair- JBJS Br.2007
5. Burkhart SS, De Beer JF. Traumatic glenohumeral bone defects and their relationship to failure of arthroscopic Bankart repairs: significance of the inverted-pear glenoid and the humeral engaging Hill-Sachs lesion. Arthroscopy 2000;16:677-694)

6. Franz S. Kralinger et al. :Predicting recurrence after primary anterior shoulder instability Am J Sports Med 2002 vol.30 No 1 116-120

7. Gagey OJ, Gagey N. The hyperabduction test. J Bone Joint Surg Br. 2001;83:69-74

8. Gerber C, Ganz R. Clinical assessment of instability of the shoulder: with special reference to anterior and posterior drawer tests. J Bone Joint Surg Br 1984;66:551-6

9. Itoi E, Lee SB, Amrami KK et al (2003) Quantitative assessment of classic anteroinferior bony Bankart lesions by radiography and computed tomography. Am J Sports Med 31:112-118

10. Itoi E, Lee SB, Berglund LJ et al (2000) The effect of a glenoid defect on anteroinferior stability of the shoulder after Bankart repair: a cadaveric study. J Bone Joint Surg Am 82:35-46

11. Jobe F, Kvitne RS. Shoulder pain in the overhand or throwing athlete: the relationship of anterior instability and the rotator cuff impingement. Orthop Rev 1989;18:963-75)

12. Lo I, Parten P., Burkhart S. The Inverted Pear Glenoid: An Indicator of Significant Glenoid Bone Loss Arthroscopy: The Journal of Arthroscopic and Related Surgery, Vol 20, No 2 (February), 2004: pp 169-174

13. Milano C. et al.: Analysis of risk factors for glenoid bone defect in anterior shoulder instability Am J Sports Med 2011 Sep;39 (9) 1870-6

14. Rowe CR, Patel D, Southmayd WW. The Bankart procedure: a long-term end-result study. J Bone Joint Surg Am 1978; 60:1-16

15. Sugaya H, Moriishi J, Dohi M (2003) Glenoid rim morphology in recurrent anterior glenohumeral instability. J Bone Joint Surg Am 85:878-884

16. Sugaya $\mathrm{H}$, Moriishi J, Dohi M, Kon Y, Tsuchiya A. Glenoid rim morphology in recurrent anterior glenohumeral instability. J Bone Joint Surg Am

17. Yamamoto $\mathrm{N}$, Itoi $\mathrm{E}$, Abe $\mathrm{H}$, et al. Contact between the glenoid and the humeral head in abduction, external rotation, and horizontal extension: A new concept of glenoid track. J Shoulder Elbow Surg 2007;16:649-656.15 
Association between the Risk Factor for Anterior Glenohumeral Instability and Bone Loss in Active Sport Players

18. Yamamoto N, Itoi E, Abe H, et al. Effect of an anterior glenoid defect on anterior stability: a cadaveric study. Am J Sports Med. 2009;37:949-54

19. Yamamoto N, Muraki T, Sperling JW, et al. Stabilizing mechanism in bone-grafting of a large glenoid defect. J Bone Joint Surg. 2010;92:2059-66 